$\mathcal{S}_{\text {doi: } 10.3765 / \text { sp.4.3 }}^{\text {Semantics \& Pragmatics Volume 4, Article 3: 1-43, } 2011}$

\title{
Explaining presupposition projection with dynamic semantics*
}

\author{
Daniel Rothschild \\ All Souls College, Oxford
}

Received 2011-03-07 / First Decision 2011-04-09 / Revision Received 2011-04-20 /

Second Decision 2011-06-o9 / Revision Received 2011-06-15 / Published 2011-07-07

\begin{abstract}
Heim's (1982, 1983b) dynamic semantics provides an attractive system for capturing the basic facts about presupposition projection. A longstanding criticism of this semantics is that it requires for each connective lexical stipulations that are not determined by its truth-conditional meaning. I give a precise formulation of this criticism in terms of what I call a rewrite semantics. Then, I use this idea of a rewrite semantics to formulate a new version of dynamic semantics. This version does not require stipulations particular to individual connectives, but rather allows a derivation of the presupposition projection properties for each connective from its truthconditional meaning.
\end{abstract}

Keywords: presupposition projection, dynamic semantics, rewrite rules

\section{Introduction}

Consider these three sentences:

a. John stopped smoking.

b. If John used to smoke, then John stopped smoking.

c. Either John didn't use to smoke, or he stopped smoking.

* This paper grew out of an earlier paper entitled "Making Dynamic Semantics Explanatory". I am grateful to Be Birchall, Haim Gaifman, Nathan Klinedinst, James Shaw, Benjamin Spector and workshop audiences at the ENS and the University of Chicago for their helpful comments. Emmanuel Chemla and Philippe Schlenker deserve special thanks. This paper changed greatly - for the better - in response to many comments, criticisms and suggestions from three anonymous referees for $S \& P$ as well as the editors-in-chief there, Kai von Fintel and David Beaver.

(C)2011 Daniel Rothschild

This is an open-access article distributed under the terms of a Creative Commons NonCommercial License (creativecommons.org/licenses/by-nc/3.0). 
Sentence (1a) presupposes that John used to smoke but neither sentence (1b) nor sentence (1c) does. ${ }^{1}$ This is an instance of the pattern of presupposition projection, the way complex expressions inherit or, as in this case, fail to inherit the presuppositions of their parts. Ideally, there would be something relatively simple we could say about why (1b) and (1c) don't give rise to the presupposition that (1a) does. Saying it has turned out to be surprisingly difficult.

The matter is a bit confused by the fact that there is one thing we can say that sounds quite nice and covers this small set of data, but that doesn't generalize. According to Gazdar (1979) the reason (1b) and (1c) don't presuppose that John used to smoke, is that these presuppositions are not consistent with the conversational assumptions necessary for (1b) and (1c) to be appropriate utterances. Indeed, it does seem that (1b) and (1c) would be rather odd things to say in contexts in which we already took for granted that John used to smoke. The problem with this story is that when we slightly modify ( $1 \mathrm{~b})$ and (1c) so that they no longer have this feature, the presuppositions do not magically reappear: ${ }^{2}$

(2) a. If John used to smoke heavily, then John stopped smoking.

b. Either John didn't use to smoke heavily, or he stopped smoking.

So the simple conversational-condition strategy of explaining presupposition projection fails.

Gazdar's account, though inadequate, does have the virtue that it gives a clear explanation for the pattern in (1a) to (1c) without appeal to any special notions about the meanings of the connectives appearing in those examples (i.e. if ... then ... and or).

Heim's paper "On the Projection Problem for Presuppositions" (1983b) proposed a replacement of truth-conditional semantics with a dynamic semantics that treats meanings as instructions to update the common ground. One of the selling of points of Heim's "dynamic semantics", as it has come to be known, was that predictions about the pattern of presupposition projection seemed to fall out of the system. A major objection to this way of explaining presupposition projection is that the treatment of binary connectives is not explanatory (Soames 1982, Heim 1990, Schlenker 2008a): Heim needs to stipulate the presupposition projection properties for each binary connective rather than use one over-arching principle as Gazdar did.

1 I am assuming a basic familiarity with the notion of presupposition as currently used within the semantics community. See e.g. Soames 1989, Beaver 2001, and Beaver \& Geurts 2011.

2 This observation is due to Soames (1982). 
Explaining presupposition projection

In this paper I show that a modification of Heim's account yields the same predictions without recourse to stipulations peculiar to individual connectives or quantifiers. My modification of Heim's account goes roughly as follows: Heim defined the meaning of connectives and quantifiers by means of rewrite rules that allow one to state the truth conditions for complex dynamic formulas in a language using only set theory and simple dynamic formulas. Heim assigned a separate rewrite rule for each binary connective and her stipulations are located in the details of each of these rules. I suggest that we can use a semantics where sentences are defined iff there exists some rewrite rule for the connective that both gets the truth-conditions correct and does not lead to a presupposition failure. This account, once a simple order constraint is added, yields the same predictions about presupposition projection as Heim's dynamic semantics does. Thus, I do not think there is any inherent problem with the lack of explanatory power in dynamic semantics, and, the choice between dynamic semantics and other approaches to presupposition projection needs to rest on other criteria. ${ }^{3}$

Here is the plan of the paper. In §2, I introduce Stalnaker's account of assertion as an update of the common ground and the Karttunen/Stalnaker treatment of presuppositions as conditions on the common ground. (You can skip if you are already familiar with this.) §3 is a presentation of Heim’s dynamic semantics and a discussion of its handling of presupposition projection. (You should glance at the notation here even if you are familiar with dynamic semantics.) §4 and §5 form the core of the paper: In §4, I discuss the explanatory challenge to dynamic semantics and introduce the apparatus of rewrite rules. In $\S 5$, I use rewrite rules to define a loose version of dynamic semantics that reproduces the predictions about presupposition projection from dynamic semantics without individual stipulations for connectives that go beyond their truth conditions. In §5, I also define a predictively different version of dynamic semantics that is not sensitive to order. In §6, I go through the standard binary connectives, assessing the predictions of the two semantics presented here and relating these predictions to the literature. In $\S 7$, I extend the semantics above to include quantifiers, and, in §8, I discuss some empirical problems with presupposition projection under quantification.

3 This paper is largely in response to the recent criticism of dynamic semantics put forward by Schlenker (2006, 2008a). Schlenker (2009) also tries to rehabilitate dynamic semantics in a way that answers the explanatory worry, but his theory is, I think, much further from Heim's original program than the one presented here. 
Daniel Rothschild

\section{Common grounds and projection rules}

One of the marks of linguistic presuppositions is that when a sentence presupposes a proposition an assertion of the sentence seems to take the proposition for granted. We might describe presuppositions by saying that a sentence, $S$, presupposes a proposition, $p$, when an assertion of $S$ is only felicitous in a context in which the mutual assumptions of the conversational participants include $p$. This definition, due to Stalnaker (1974) and Karttunen (1974), takes linguistic presupposition to give rise to acceptability conditions on the common ground, the collection of mutually accepted assumptions among conversational participants.Q

Here is a more careful description of the framework: in a conversation any utterance is made against the common ground, which we model as the set of worlds not ruled about by the mutual assumptions of the conversational participants. When one asserts a proposition, $a$, the normal effect, if the audience accepts the assertion, is the removal of the worlds where $a$ is false from the common ground. One way of working presuppositions into this framework is to assume that certain sentences are such that they are only felicitously asserted in certain common grounds. In particular, we say that if a sentence $A$ presupposes $\underline{a}$, then $A$ is only felicitously assertable in a common ground $c$ if $c$ entails $\underline{a}$, i.e., $\underline{a}$ is true in every world in $c$ (which we write as $c \vDash \underline{a}$ ). When it is felicitous, the effect of an assertion of $A$ is to remove certain worlds from the common ground.

In this framework, due to Stalnaker and Karttunen, the projection problem is the problem of defining what conditions complex sentences put on the common ground in terms of what conditions their parts do. Below are some sample rules we could use to describe the projection behavior in this framework:
a. $b \wedge A$ is acceptable in $c$ iff $c \vDash b \rightarrow \underline{a}$
b. $b \vee A$ is acceptable in $c$ iff $c \vDash \neg b \rightarrow \underline{a}$
c. $b \rightarrow A$ is acceptable in $c$ iff $c \vDash b \rightarrow \underline{a}$

We can apply these rules to examples such as the following:

(4) a. John used to smoke and he's stopped.

b. John didn't use to smoke, or he's stopped

c. If John used to smoke, then he's stopped.

According to the rules (3a) to (3c) the presuppositions in sentences (4a) to (4c) are trivial. For instance, the presupposition of (4a) by rule (3a) is if John 
Explaining presupposition projection

used to smoke, then he used to smoke. Since this is trivially true the entire sentence is correctly predicted not to presuppose anything.

These rules can be elaborated into general rules that predict the presupposition of any complex sentence, given the presuppositions of its parts. Such a set of rules would essentially be the filtering rules developed by Karttunen (1973). There is some debate over the empirical merits of these rules, but I want to put this aside here. ${ }^{4}$

Suppose rules along the lines of (3a) to (3c) suffice to describe the pattern of presupposition projection. Merely stating these rules fails entirely to explain why the pattern of presuppositions project can be so described. Heim 1983b was a landmark paper partly because it gave a semantics of presuppositional expressions (and complexes formed out of these) from which these rules of presupposition projection follow. I will outline her account and discuss a major criticism of it, due to Scott Soames and Mats Rooth. They argued that Heim's semantics has features which effectively amount to stipulations of presupposition projection properties (Soames 1982, Heim 1990, Schlenker 2008a).

\section{Dynamic semantics}

I am going to present Heim's propositional dynamics semantics in a nonstandard way, as this will facilitate some of the later discussion. ${ }^{5}$ While the basic ideas may be familiar to some readers, it is worth skimming through this section to get a sense of the notation.

The major change in Heim's dynamic semantics, from the Stalnakerian framework discussed above, is that the meanings of sentences are no longer propositions, sets of possible worlds, but instead ways of changing the common ground. Thus, a sentence has as its semantic value a function from sets of possible worlds to sets of possible worlds (i.e. a function with domain $\mathcal{P}(W)$ and range $\mathcal{P}(W))^{6}$

Using this kind of semantic value we can reproduce the Stalnakerian treatment of assertion. Instead of having a sentence $S$ denote a set of possible

4 There is a long tradition that argues against these conditional presuppositions (most notably Geurts 1996).

5 There are many changes from Heim's original paper (1983b). Most significantly: the notation is more in line with contemporary usage, presuppositions are modeled explicitly as partially defined functions, and letters representing contexts are brought into the object language.

6 Notation: $W$ denotes the set of all possible worlds, and for any set $X, \mathcal{P}(X)$ denotes the set of all subsets of $X$, i.e. the powerset of $X$. 
worlds $p$ we have the sentence denote the function that goes from a common ground $c$ to the intersection of $p$ and $c$. In other words, a sentence denotes a function that captures what it is to update any common ground with the sentence. Heim named this sort of function a context change potential, or CCP for short.

Much of the allure of dynamic semantics, in particular the treatment of donkey anaphora, comes from its treatment of variables which a propositional fragment cannot capture. However, most of Heim's treatment of presupposition projection can be expressed in a propositional fragment. For now I will only discuss the propositional case, and introduce variables and quantifiers later in $§ 7$.

Presuppositional meanings are encoded by partial functions from contexts to contexts. Consider a sentence like John stopped smoking. In a classical semantics we would assign this sentence as its meaning the set of possible worlds where John used to smoke and doesn't any more. However, in a partial, dynamic semantics we assign this sentence a partial function, $f: \mathcal{P}(W) \rightarrow \mathcal{P}(W)$, such that:

- $f(x)$ is defined iff John used to smoke in all worlds in $x$

- where defined $f(x)=\{w \in x$ : John no longer smokes in $w\}$.

Since John stopped smoking is not defined when the context does not entail that John used to smoked, it is infelicitous in such a context. Thus, the partiality of the CCPs captures their presuppositional behavior.

It is helpful to note that Heim's treatment of presuppositions as partially defined CCPs is technically similar to the older tradition of modeling presuppositions with a trivalent semantics. On a trivalent semantics each sentence can be true in some worlds, false in others, and undefined in others. Typically, we say that if a sentence $S$ has a presupposition failure, then it is neither true nor false. So John stopped smoking has the following truth-condition:

- John stopped smoking is true iff John used to smoke and he doesn't any longer.

- John stopped smoking is false iff John used to smoke and he still smokes.

- John stopped smoking is neither true nor false iff John didn't used to smoke. 
Explaining presupposition projection

Stalnaker (1973) proposed that the following pragmatic rule should govern the assertion of such sentences: ${ }^{7}$

(5) Only assert a trivalent sentence $S$ in a common ground $c$, if $S$ is true or false in every world in $c$.

If we followed this rule, then $S$ could only result in a felicitous update of $c$ if $c$ entails that $S$ is either true or false. Thus, a trivalent semantics does the same basic thing a CCP semantics does: it formally encodes the presuppositions of sentences in terms of definedness conditions.

So far, dynamic semantics looks like a different technical framework for expressing what a trivalent semantics does. The interest comes when we introduce the compositional rules for complex sentences. Before we do that, however, we need to state the details of the semantics in a more precise way. We could simply give a semantics for sentences which assigns as values not propositions, but CCPs. However, to facilitate the later discussion, I will introduce a language that includes not just CCPs but also formulas representing contexts or common grounds. So this language will include two parts: 1) the context part for formulas representing common grounds and 2) the CCP part for sentences expressing context change potentials. Properly speaking, then, the only part of the formal language that corresponds to the actual spoken (or written) language is the CCP-part. However, this is just a notational convenience, not itself a substantive assumption.

\subsection{Syntax}

- lower-case letters $a, b, c \ldots$ are atomic sentences (these will be used to model contexts)

- upper-case letters $A, B, C \ldots$ are atomic CCPs (these represent sentences in human language)

- the set of CCPs is defined as follows:

- any atomic CCP is a CCP

- if $\phi$ and $\psi$ are CCPs then so are $\neg \phi, \phi \wedge \psi, \phi \vee \psi$, and $\phi \rightarrow \psi$

7 See Soames 1989 for an interesting criticism of this pragmatic rule. Soames's most important point is that if we use trivalence to capture vagueness as well as presupposition failure, this rule predicts that a vague sentence has non-trivial presuppositions. Soames argues convincingly that this is a bad prediction. 
- the set of complex sentences is defined as follows:

- any atomic sentence is a sentence

- if $\alpha$ and $\beta$ are sentences then so are $\alpha \wedge \beta, \alpha \vee \beta$, and $\alpha \backslash \beta^{8}$

- if $\alpha$ is a sentence and $\phi$ is a CСP then $\alpha[\phi]$ is a sentence

As noted, the actual complex sentences in this language represent contexts including ones which are combined or updated by CCPs in various ways. So, for instance, $c[A \wedge B]$ represents the update of the context $c$ by the complex CCP $A \wedge B$. It may seem that syntactic rules for combining contexts to get such formulas as $a \wedge b$ are pointless since contexts themselves are not syntactic objects, but they will come in handy for giving the semantics of complex CCPs.

\subsection{Partial semantics}

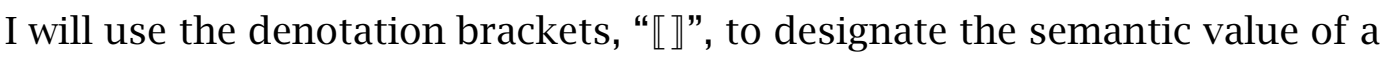
sentence or a CCP. An interpretation I sets the semantic values for both the atomic sentences and the atomic CCPs, while the semantic values of complex formulas is given by recursive semantic rules. For every atomic sentence $\alpha$, $\llbracket \alpha \rrbracket_{I}$ is a set of possible worlds (i.e. a subset of $W$ ). For every atomic CCP $\alpha$, $\llbracket \alpha \rrbracket_{I}$ is a function from sets of possible worlds to sets of possible worlds (i.e. from $\mathcal{P}(W)$ to $\mathcal{P}(W))^{9}$

The semantic value of all complex sentences, as we will see, are sets of possible worlds. A sentence $\alpha$ entails $\beta$ (which we write $\alpha \vDash \beta$ ) iff on every interpretation, $I, \llbracket \alpha \rrbracket_{I} \subseteq \llbracket \beta \rrbracket_{I}$. The semantic value of all complex CCPs, which we will define later, are functions from $\mathcal{P}(W)$ to $\mathcal{P}(W)$; we will not need entailment relations for these.

Let us first discuss the semantic value of sentences of the form $\alpha[\psi]$ where $\alpha$ is a sentence and $\psi$ is an atomic CCP. Our semantic rule for CCP application is functional application:

$$
\llbracket \alpha[\psi] \rrbracket_{I}=\llbracket \psi \rrbracket_{I}\left(\llbracket \alpha \rrbracket_{I}\right)
$$

8 Throughout, I assume and suppress when unnecessary standard parenthetical notation to mark order of operations.

9 Hence $I$ can be specified by a triplet, $\langle W, S, C\rangle$, where $W$ is a set of possible worlds, $S$ is a function from atomic sentences to subsets of $W$, and $C$ is a function from atomic CCPs to functions from $\mathcal{P}(W)$ to $\mathcal{P}(W)$. I will often suppress mention of $I$. 
Explaining presupposition projection

We assume (naturally) that, $\llbracket \alpha[\psi] \rrbracket_{I}$ is defined iff both $\llbracket \psi \rrbracket_{I}$ and $\llbracket \alpha \rrbracket_{I}$ are defined and the latter is in the range of the former. (Since presuppositions arise because of undefinedness, such assumptions matter.)

We will also give recursive semantic rules for the connectives when they apply to sentences. ${ }^{10}$ These are as expected:
a. $\llbracket \alpha \wedge \beta \rrbracket_{I}$ is $\{w: w \in \llbracket \alpha \rrbracket$ and $w \in \llbracket \beta \rrbracket\}$
b. $\llbracket \alpha \vee \beta \rrbracket_{I}$ is $\{w: w \in \llbracket \alpha \rrbracket$ or $w \in \llbracket \beta \rrbracket\}$
c. $\llbracket \alpha \backslash \beta \rrbracket_{I}$ is $\{w: w \in \llbracket \alpha \rrbracket$ and $w \notin \llbracket \beta \rrbracket\}$

We assume here that for an arbitrary binary connective $*, \llbracket \alpha * \beta \rrbracket$ is defined iff both $\llbracket \alpha \rrbracket$ and $\llbracket \beta \rrbracket$ are defined. (This is the standard, week-Kleene treatment of combinations of partially defined formula, something that is only implicit in Heim's original papers.)

This semantics does not cover the entire language since we have only given a semantics for sentences formed without the use of the recursive syntax for CCPs: we have no way of handling any formula that includes a complex CCP such as $a[A \wedge B]$ or $a[\neg A]$. Complex CCPs is where the action is: I turn now to Heim's treatment.

\subsection{Semantics of complex CCPs}

On my formulation of Heim's semantics for complex CCPs, which is very close to her original treatment, their meaning is defined recursively in terms of the semantics of the language already given.

(8) If $\alpha$ is a sentence and $\phi$ and $\psi$ are CCPs then:
a. $\alpha[\neg \phi]=\alpha \backslash \alpha[\phi]$
b. $\alpha[\phi \wedge \psi]=(\alpha[\phi])[\psi]$
c. $(\alpha[\phi] \vee \psi)=\alpha[\phi] \vee(\alpha[\neg \phi])[\psi]$
d. $(\alpha[\phi] \supset \psi)=\alpha[\neg \phi] \vee(\alpha[\phi])[\psi]$

Note: the equal sign is used to designate equality of semantic value, not syntactic equality, semantic evaluation brackets here and later are suppressed for readability.

10 Despite using the same symbols for connectives joining sentences and connectives joining CCPs, these are different connectives with different semantics. 
Daniel Rothschild

\subsection{Assessment}

These rules complete the semantics for the language as repeated applications of them will yield an interpretation of any formula. Two main properties recommend this semantics for complex CCPs:

i. It gets the truth conditions of complex sentences correct.

ii. The rules of presupposition projection fall out of it.

To see the way in which the proposal gets the truth-conditions right requires looking at an example of a complex CCP. Consider the CCPs we should assign to It stopped raining, $R$, and John is tall, $J$ :

- $\llbracket R \rrbracket=$ function $f$ s.t. $f(p)$ is defined if and only if $p$ is a set of possible worlds in all of which it used to rain, and when defined $f(p)=p \cap\{w \in p$ : it doesn't rain now in $w\}$

- $\llbracket J \rrbracket=$ function $g$ that takes any set of possible worlds $p$ and returns $\{w \in p:$ John is tall in $w\}$

Now we can ask what happens when we update a context $c$ with the complex CCP $R \wedge J$. Applying (8b) we get: $c[R \wedge J]=(c[R])[J]=g(f(c))$. When defined $g(f(c))=\{w \in c$ : it doesn't rain now in $w$ and John is tall in $w\}$. When defined, this is exactly the context you would get when you update it with the propositions that it stopped raining and that John is tall. So the rule for conjunction allows complex CCPs to mimic the effect on the common ground of adding complex sentences in a classical semantics. Similar remarks apply to the other definitions above (as long as we understand the conditional as the material conditional).

With regard to point (ii) above, the question is what the definedness conditions of complex CCPs are in terms of the definedness conditions of their parts. Each of Heim's semantic rules in (8) uniquely determines a definedness condition. Using her rule for conjunction, $\alpha[\phi \wedge \psi]=(\alpha[\phi])[\psi]$, we can see that $c[R \wedge J]$ is defined iff $(c[R])[J]$ is defined which it is iff $g(f(c))$ is defined. Given that $f$ is a partial function and $g$ is a total function, the only way this can fail to be defined is if $f(c)$ is not defined. By definition $f(c)$ is defined iff $c$ only includes worlds where it used to rain. This matches the predictions of standard accounts: for the sentence to not have a presupposition failure $c$ must include the information that it is raining. If we switch the 
Explaining presupposition projection

order of $R$ and $J$ we get the standard Karttunen prediction that $c[J \wedge R]$ is defined if and only if in every world in $c$ in which John is tall it used to rain.

If we work through the predictions for all the connectives we get standard predictions, ones that capture the generalizations in $\S 2$. I will call the generalizations about presupposition projection that follow from Heim's definedness conditions the Karttunen/Heim projection rules. ${ }^{11}$

\section{Explanatory challenge}

A persistent criticism of Heim's program concerns the relationship between points (i) and (ii) above. Although Heim does not directly say so, it is clear that she thought that her dynamic framework had the property that once you assign truth-conditionally adequate semantics for complex expressions, the Karttunen rules for presupposition projection will follow. In other words, Heim thought that given the syntax and the partial semantics above, the only way of extending the semantics to cover complex CCPs would be in a way that would yield the Karttunen/Heim projection rules. ${ }^{12}$

In its strongest interpretation this claim is trivially false. Given the partial semantics for CCPs in §3, there are numerous possible ways we could extend the semantics to handle complex CCPs. Some of them will result in the disappearance of all standard presuppositions when the presuppositional trigger appears in a complex clause. Consider, for instance, this rule for handling conjunctive CCPs:

(9) $\quad \alpha[\phi \wedge \psi]$ is defined iff there is largest subset $a$ of $\llbracket \alpha \rrbracket$ such that $\llbracket \psi \rrbracket(\llbracket \phi \rrbracket(a))$ is defined. If it is defined, $\alpha[\phi \wedge \psi]=\llbracket \psi \rrbracket(\llbracket \phi \rrbracket(a))$

11 This label is not very accurate for disjunction: Karttunen, in fact, made different predictions, while Heim (1983b) does not discuss the case of disjunctions. I discuss disjunction further in $§ 6.2$.

12 She wrote about the quantifier 'no': "Here as elsewhere, the theory I am advocating gives me no choice: Once I have assigned 'no' a CCP that will take care of its truth-conditional content, it turns out that I have to side with Cooper [about the presupposition projection properties of 'no']." It is clear from her later writing that she thought this applied also to the binary connectives so that, in some sense, the projection properties follow from the truth-conditional meaning. Heim (1990) writes "In my 1983 paper, I was less cautious than Karttunen or even Stalnaker and claimed that if one only spelled out the precise connection between truthconditional meaning and rules of context change, one would be able to use evidence about truth conditions to determine the rules of context change, and in this way motivate those rules independently of the presupposition projection data that they are supposed to account for." 
The effect of this cumbersome CCP, in Heim's terminology, is to locally accommodate the presuppositions of $\phi$ and $\psi$. In other words, its effect is to strengthen the sentential variable $\alpha$ used to calculate the meaning to make sure that the calculation does not fail, if there is a unique way to do so. Heim herself introduces local accommodation as the way of understanding cases in which presuppositions fail to appear - what was previously called "cancellation" in the presupposition literature. So, it is clear from her paper that there are ways of treating the semantics of complex expressions that do not capture Karttunen's projection rules. We can conclude that with no restrictions at all on how to determine the meaning of complex CCPs we do not predict anything about their projection properties by just using the partial dynamic semantics outlined in §3.

If we look at Heim's actual semantics for the different connectives, repeated below, we see that all of her semantic rules have roughly the same form.

$$
\begin{aligned}
& \text { a. } \alpha[\neg \phi]=\alpha \backslash \alpha[\phi] \\
& \text { b. } \alpha[\phi \wedge \psi]=(\alpha[\phi])[\psi] \\
& \text { c. }(\alpha[\phi] \vee \psi)=\alpha[\phi] \vee(\alpha[\neg \phi])[\psi] \\
& \text { d. }(\alpha[\phi] \supset \psi)=\alpha[\neg \phi] \vee(\alpha[\phi])[\psi]
\end{aligned}
$$

These rules are all simpler than the local-accommodation rule in (9). We might think that Heim's claim in her paper was that any semantics for complex CCPs of this sort would have the right presupposition projection properties.

To make this claim precise we need to give a characterization of the sort of semantic rules Heim employs. Her semantics for complex CCPs are all what I call rewrite rules: they specify how to rewrite formulas with complex CCPs into formulas without them. If we limit the possible semantics for complex CCPs to those expressible with such rewrite rules, we would eliminate (9) as a possible semantic rule for conjunction.

On a natural construal of Heim's paper her implicit claim is that any rewrite rule which correctly captures the truth conditions for a sentence will yield Karttunen's rules for presupposition projection. ${ }^{13}$ Understood this way the possibility of a semantics for conjunction like (9) does not refute Heim's

13 In fact it is difficult to know what Heim had in mind, since there is no precise claim in the paper along these lines, and whatever she thought, as she later acknowledged, was wrong. So any interpretation of her paper will, by necessity, seem somewhat uncharitable. I choose this interpretation since it makes sense of the Soames and Rooth objection, which Heim (1990) admitted as valid. 
Explaining presupposition projection

claim. What matters rather is what semantics for complex CCPs expressible as rewrite rules are possible.

However, Scott Soames (1989) and Mats Rooth independently made observations that show that even a rewrite semantics for complex CCPs need not get the facts for presupposition projection right. ${ }^{14}$ There are many "deviant" rewrite semantics for complex CCPs that match the truth-conditions of Heim's but have different definedness conditions. For example the following rule is often cited as a deviant rule for conjunction:

$$
\alpha[\phi \wedge \psi]=(\alpha[\psi])[\phi]
$$

When defined, this rule will always do what normal conjunction does (in a sense to be made precise below). However, its definedness conditions are different from Heim's rule for conjunction. It is defined if and only if the second conjunct is defined in the starting context $\alpha$ and the first conjunct is defined when applied to the result of updating the $\alpha$ with the second conjunct. This is the opposite of the normal prediction. According to rule (11), the following sentence should have no presuppositions:

(12) Mary knows John is tall and John is very tall.

On the Karttunen/Heim rules, however, (12) presupposes that John is tall. So, in fact, merely limiting oneself to a dynamic semantics based on rewrite rules of the kind Heim uses does not determine the rules of presupposition projection.

It will be useful to be even more explicit about this reconstruction of Heim's implicit claim and Soames and Rooth's objection to it. There are really two notions that need to be spelled out: 1) the class of rewrite rules that could give the semantics for complex CCPs and 2) what it means for a rewrite semantics to be truth-conditionally adequate to standard conjunction, disjunction, and so forth.

\subsection{Rewrite rules}

I will use $*$ to represent an arbitrary binary connective. Let $\alpha$ be a sentence and $\phi$ and $\psi$ be CCPs. A rewrite rule for $\alpha[\phi * \psi]$ is is any formula formed out of $\alpha, \phi$, and $\psi$ using all the standard rules of syntactic composition from $\S 3$ except insofar as no new atomic formulas or CCPs may be added and no

14 Rooth's observation is in a 1987 letter to Heim which she quotes in her 1990 paper. 
rules for forming complex CCPs may be used. We can state this as a recursive definition of a class of formulas:

(13) For any sentence $\alpha$, CCPs $\phi$ and $\psi$, and binary connective $*$ :

a. $\alpha$ is a rewrite rule for $\alpha[\phi * \psi]$

b. if $\beta$ is a rewrite rule for $\alpha[\phi * \psi]$ then so are $\beta[\phi]$ and $\beta[\psi]$

c. if $\beta$ and $\gamma$ are rewrite rules for $\alpha[\phi * \psi]$ then so are $\beta \wedge \gamma, \beta \vee \gamma$, and $\beta \backslash \gamma$.

We can generalize this notion to include rewrite rules for formulas with complex CCPs with negation, such as $\alpha[\neg \phi]$. A rewrite rule for a sentence of the form $\alpha[\neg \phi]$ is the same as above except we can only use the CCP $\phi$ in forming it.

Technically a rewrite rule for a sentence $\alpha[\phi * \psi]$ is just another sentence. Why do we call it a rule? Well, consider again Heim's semantics for conjunction:

(14) $\quad \alpha[\phi \wedge \psi]=(\alpha[\phi])[\psi]$

On this semantics the complex CCP on the left-hand side of the equality has its meaning defined by the rewrite rule on the right-hand side. We will say that a semantic rule for a connective $*$ is a rewrite semantics if it is statable as $\alpha[\phi * \psi]=\gamma$, where $\gamma$ is a rewrite rule for $\alpha[\phi * \psi]$. It is easy to see that all of Heim's rules for complex CCPs, in (8), are rewrite semantics. ${ }^{15}$

Note, however, that the notion of a rewrite semantics for a complex CCP does not depend on how a semantic rule is stated, but only on its actual content. Nonetheless, in some cases it is easy to see that a given semantics is not statable as a rewrite rule. For example, the local-accommodation semantics in (9) above is not statable as a rewrite rule, so is not a rewrite semantics. ${ }^{16}$

\subsection{Truth-conditional adequacy}

Intuitively, truth-conditional adequacy is the property that Heim's original rule, $(8 \mathrm{~b})$, and the deviant rule, (11), for conjunction share: they capture

15 Note that one semantics may be expressible by more than one semantically identical but syntactically distinct rewrite rule: a semantics does not uniquely determine a rewrite rule.

16 This fact is quite intuitive, though giving a proof is not entirely trivial. The proofs in the appendix will give the reader a sense of this can be proved by induction over the class of rewrite rules. 
Explaining presupposition projection

the meaning of standard truth-conditional conjunction. What we need to determine the truth-conditional adequacy of a given rewrite rule is a way of telling whether the update it defines is equivalent to the update that a classical semantics would give.

The basic procedure to check truth-conditional adequacy goes as follows. Suppose $\gamma$ is a rewrite rule for $\alpha[\phi * \psi]$. First, we replace each instance in $\gamma$ of $\alpha, \phi$ and $\psi$, with arbitrary formulas of classical logic (say, $a, p$ and $q$, respectively). Then, we substitute conjunction for applications of CCPs as well as substituting $\wedge \neg$ for $\backslash$. The product of these syntactic transformations is a well-formed formula in classical logic. Last, we check if the formula resulting from these transformations is logically equivalent to a standard classical update for the connective being defined, which is $a \wedge(p * q)$. If it is equivalent, the rule is truth-conditionally adequate, otherwise it is not.

Here's an example: Consider Heim's rule for conjunction, $\alpha[\phi \wedge \psi]=$ $(\alpha[\phi])[\psi]$. The right-hand side is a rewrite rule. We can syntactically transform the rewrite rule into propositional logic by substituting $a$ for $\alpha$, $p$ for $\phi$, and $q$ for $\psi$. We also replace all instances of CCP-applications with conjunction. This procedure allows the following transformation:

$$
(\alpha[\phi])[\psi] \Rightarrow(a[p])[q] \Rightarrow(a \wedge p) \wedge q
$$

Classically, if we think of $a$ as a propositional sentence true only in the common ground, then the result of updating this common ground with $p \wedge q$ is the worlds where $a \wedge(p \wedge q)$ is true. As this last formula is logically equivalent to the result of the transformation in (15), $(a \wedge p) \wedge q$, Heim's rule for conjunction is truth-conditionally adequate.

Here is a more precise, general statement of this way of determining whether a rewrite rule is truth-conditionally adequate.

(16) For any sentence $\alpha$, and CCPs $\phi$ and $\psi$, a rewrite rule $\gamma$ for $\alpha(\phi * \psi)$ is truth-conditionally adequate iff for arbitrary sentences $a, p$, and $q$, $a \wedge(p * q)$ is logically equivalent to $\gamma^{\prime}$ where $\gamma^{\prime}$ is the result of the making the following syntactic changes to $\gamma$ :

a. for any sentence $\beta$, and any CCP, $\tau$, replace $\beta[\tau]$ with $\beta \wedge \tau$

b. replace every instance of $\alpha$ with $a, \phi$ with $p, \psi$ with $q$, and $\backslash$ with $\wedge \neg$.

Corresponding to this definition of truth-conditional adequacy for a rewrite rule is a definition of truth-conditional adequacy for a rewrite semantics: A rewrite semantics for $*$ expressible as $\alpha[\phi * \psi]=\gamma$, where $\gamma$ is a rewrite 
rule for $\alpha[\phi * \psi]$, is truth-conditionally adequate if and only if $\gamma$ is a truthconditionally adequate rewrite rule for $\alpha[\phi * \psi]$.

Here are some examples of truth-conditionally adequate rewrite semantics for $\vee$ according to these definitions:

$$
\begin{array}{ll}
\text { a. } \alpha[\psi \vee \phi]=\alpha[\phi] \vee \alpha[\psi] \\
\text { b. } \alpha[\psi \vee \phi]=\alpha \backslash((\alpha \backslash \alpha[\phi]) \backslash(\alpha \backslash \alpha[\phi])[\psi])
\end{array}
$$

These are not truth-conditionally adequate rewrite semantics, however:

$$
\begin{aligned}
& \text { a. } \alpha[\psi \vee \phi]=\alpha[\phi] \wedge \alpha[\psi] \\
& \text { b. } \alpha[\psi \vee \phi]=\alpha \backslash(\alpha \backslash \alpha[\phi])[\psi]
\end{aligned}
$$

With this explicit understanding of rewrite rules and truth-conditional adequacy we can now state a version of what I suggested above may have been Heim's implicit claim about the explanatory power of her semantics:

(19) For any given connective $*$, any two truth-conditionally adequate rewrite semantics for $*$ will determine the same presupposition projection properties, which are the properties outlined by Karttunen (for, at least, conjunction, conditionals, negation).

Once it is stated baldly we can easily see that the conjecture is false by using the deviant rewrite rule for conjunction (11) suggested by Soames and Rooth. This rewrite rule is truth-conditionally adequate for disjunction but has different projection properties from the Karttunen/Heim rule. ${ }^{17}$

In this way, the basic framework of dynamic semantics combined with the idea of a rewrite semantics fails to give a system sufficiently constrained to predict the pattern of presupposition projection. This, of course, does not show that the framework is wrong. But it does leave the theory needing a separate stipulation for the semantics of each binary connective. If there were only three connectives this might not seem such a bad situation, but, of course, there are a host of other constructions with different presupposition-projection behavior besides and, or and if that a theory should make predictions about. These include quantifiers and other connectives like unless and because. Ideally, we want to find generalizations that explain whatever pattern of presupposition projection is observed across all these different expressions. ${ }^{18}$

17 Note that the problem is not essentially about the order of the arguments: (17a) also has different projection properties, but shares the same order of arguments as Heim's rule does. 18 Of course, we could make these generalizations at the level of the lexicon. That is they could be similar in status to Horn's (1972) generalizations about the the lack of nand (i.e. a single lexical item for not and) across languages (see also Levinson 2000). 
Explaining presupposition projection

\section{Explanatory approaches}

It is worth looking at, in general, what a semantics would need to do to overcome the particular form of explanatory inadequacy which Heim's dynamic semantic suffers from. Of course, if one is giving a semantic account of presupposition projection, as Heim does, the semantics itself should yield the projection properties, and so there must be some degree of stipulation. However, what Heim wanted was a semantic system according to which any connective with a given truth-conditional meaning will have the same projection properties. For any semantics to have this property, it will need to include some general principles that apply to the treatment of all logical connectives. I argued that Heim's dynamic semantics suggests one such principle: the principle that semantics of compound CCPs be expressible as rewrite semantics. This cross-connective principle, however, proved to be inadequate. In order to overcome the explanatory problem, we need to formulate, within the dynamic system, a principle that predicts the facts about presupposition projection.

Explanatory theories have been developed outside the dynamic framework for deriving the basic pattern of presupposition projection. Heim (1990) gives the following suggestion for what resources one might use to explain the pattern of presupposition projection:

If we wanted to deduce at least some aspects of [Karttunen's projection rules] from deeper principles or independent evidence, in what direction should we look? Two possibilities come to mind: explore to what extent these rules are predictable from the linear order of the constituent clauses, and to what extent they might follow from facts about each connective's truthconditional meaning.

The suggestion then is to have a recipe that takes as input a connective's truth-conditional meaning and the syntactic position (or the linear order) of its arguments, and outputs its presupposition projection properties. At the time Heim wrote the quote above there were no systems that were able to derive Karttunen's rules of presupposition projection from deeper principles. Since Schlenker's work, a number of such theories have been developed, which take up exactly this suggestion. Schlenker (2006, 2008a, 2009) details two related systems that yield recipes for deriving the Karttunen results using exactly the components Heim suggested, truth-conditional meaning 
and linear order. There are other strategies: George (2007) and Fox (2008) develop trivalent systems that derive the Karttunen rules for a propositional fragment, while Chemla (2008) provides a way of deriving Karttunen's rules as a form of scalar implicature. ${ }^{19}$

There are various different ways of making a dynamic semantic system that is explanatory in the sense above. All we need to do is postulate a constraint on the space of possible semantics for compound CCPs, that has the effect that any semantics for a connective $*$ that satisfies the constraint and gets the truth-conditions right also captures the projection properties. Of course, the degree to which such a principle actually provides an explanation of the Karttunen rule will depend on how plausible (and simple) the principle itself is. For example, we gain no explanatory purchase if the constraint simply amounts, itself, to a stipulation of the Karttunen rules.

I argue here that what is in a sense the loosest possible dynamic rewrite system, in which we can use any rewrite rule that is defined to interpret complex CCPs, when combined with an order constraint will yield the Karttunen/Heim projection rules. I will present this idea in two parts: In §5.1, I introduce the loose rewrite semantics and show that it makes very similar predictions to Heim's except that the rules do not take into account order, which Heim's system does. I will argue that in many cases these predictions seem superior to hers. In §5.2, I show that when you add an order constraint for all connectives to the loose rewrite semantics, you get a system that precisely reproduces Heim's predictions.

\subsection{Loose rewrite system}

By the loosest possible rewrite system, I mean a semantics for connectives in which one is allowed to choose whichever rewrite rule works in order to satisfy the presuppositions of a given sentence. ${ }^{20}$ One way of implementing this is to give an explicit semantics for any arbitrary binary connective which allows one, in effect, to choose whichever rewrite rule is defined. It goes as follows:

19 See Schlenker 2008d for a review of these theories, including an earlier version of the current one.

20 In some ways, this can be seen as a formal implementation of Soames' (1989) suggestion for handling presupposition projection in disjunction. 
Explaining presupposition projection

(20) $\alpha[\phi * \psi]$ is defined iff a) there exists a truth-conditionally adequate rewrite rule for $\alpha[\phi * \psi]$ whose semantic value is defined and b) all such rewrite rules have the same semantic value. When $\alpha[\phi * \psi]$ is defined, its semantic value is that of the truth-conditionally adequate rewrite rules for it.

This is a liberal, anything-goes rule, that allows updating with any rewrite rule, as long as it is truth-conditionally adequate. The presupposition projection behavior yielded by this rule depends on the exact property of the CCPs in the language. Here we define (very standard) semantic properties of CCPs we can use to get sharp results out of this language.

Monotonic Definedness A CCP $\phi$ has monotonic definedness conditions, if for any sentence $\alpha$, if $\alpha[\phi]$ is defined, then for any sentence $\alpha^{\prime}$ where $\alpha^{\prime}$ is stronger than $\alpha$ (i.e. every world in $\alpha^{\prime}$ is in $\alpha$ ), $\alpha^{\prime}[\phi]$ is defined.

Intersective Meaning A CCP $\phi$ has an intersective meaning, if there exists a set of possible worlds $p$ such that for any $\alpha$ when $\alpha[\phi]$ is defined it denotes $\llbracket \alpha \rrbracket \cap p$.

Note that Heim's CCPs have these features. For example, John knows it is raining is defined in a context $c$ iff $c$ entails that it is raining in $c$ (a monotonic definedness condition) and when defined always has the effect of intersecting the context with the proposition that John knows it is raining (an intersective meaning). ${ }^{21}$

Given (20) and the two conditions above, we can prove exactly what the projection rules for the connectives are. These are given in the following result, the proof of which is in the appendix.

21 As Kai von Fintel pointed out to me these assumptions are not entirely innocuous. It is commonly claimed that the presupposition of an indicative conditional is that its antecedent is at least possible according to the context: this is a non-monotonic presupposition. So the results below, Propositions 1 and 2, do not cover cases with indicative conditionals with such presuppositions. In addition, if we use Veltman's (1996) account of epistemic modals, then epistemic modals do not have intersective meanings, in the sense defined, since Veltman's epistemic modals sometimes have no effect at all on the context and sometimes take us to the absurd context. For this reason, Propositions 1 and 2 do not cover sentences with such epistemic modals. The semantics of (20) does make predictions for such cases, they are just not covered by these results. My sense is that the predictions the system makes for indicative conditionals are reasonable, but the system might need minor modification to accommodate Veltman's modal operators (in particular we must drop the requirement in (20) that all defined rewrite rules are equivalent). 
Proposition 1. Suppose $\phi$ and $\psi$ are CCPS with monotonic definedness conditions and intersective meanings and $\alpha$ is a sentence, it follows on the semantics of (2O) that:

- $\alpha[\neg \phi]$ is defined iff $\alpha[\phi]$ is defined.

- $\alpha[\phi \wedge \psi]$ is defined iff $(\alpha[\phi])[\psi]$ or $(\alpha[\psi])[\phi]$ is defined.

- $\alpha[\phi \vee \psi]$ is defined iff $(\alpha[\neg \phi])[\psi]$ or $(\alpha[\neg \psi])[\phi]$ is defined.

- $\alpha[\phi \rightarrow \psi]$ is defined iff $(\alpha[\phi])[\psi]$ or $(\alpha[\neg \psi])[\phi]$ is defined.

The projection rules on this system differ substantially from the Karttunen/Heim rules above. The major difference lies in the fact that all binary connectives have disjunctive definedness conditions in this system, whereas only one of the disjuncts serves as the definedness condition on the Karttunen/Heim rules. This disjunctive condition makes the order of the disjuncts and conjuncts irrelevant in this system. So, in this sense, the rules in Proposition 1 might be called symmetric while the Heim/Karttunen rules are asymmetric. ${ }^{22}$

Despite a preference in the literature for asymmetric theories of presupposition projection, there are many cases which can only be handled by a symmetric theory. Usually the cases are slightly more complex than the very standard cases, but I think the judgments are relatively clear. ${ }^{23}$

The following sentences are examples where the standard asymmetric theories predict that there are presuppositions, but the symmetric version of the theory above predicts no presuppositions:

22 Similar comments actually apply to the disjunctive rule for conditionals in Proposition 1, though the definition of symmetric needs to change for conditionals which are not symmetric themselves.

23 These observations build on Schlenker's work (2008a, 2009). The reason we need to look at complex cases is there may be be independent pragmatic principles interfering with our judgments in many simple cases. For example, the reason $A \wedge \underline{a}$ is unacceptable may be that there is a prohibition against saying $A \wedge B$ if $A$ entails $B$ (but not vice versa). So, for example, as Schlenker notes, following Stalnaker, the following sort of sentence is odd:

(21) John is a practicing, accredited doctor and he has a medical degree.

Whereas the reverse order is more normal:

(22) John has a medical degree and he is a practicing, accredited doctor. 
Explaining presupposition projection

(23) If John doesn't know it is raining and it is, in fact, raining heavily, then John will be surprised when he walks outside.

(24) It is unlikely that John still smokes, but he used to smoke a lot.

(25) Either the bathroom is well hidden or there is no bathroom.

In all these cases, I find the standard judgement is that there is no presupposition perceived nor do the examples seem to be marked in a way that indicates cancellation.

\subsection{Adding an order constraint}

However, we may think that there is something right about the asymmetric rules. If we want to match the Heim/Karttunen rules exactly we need to add a constraint on what kind of rewrite rules we are allowed to use. The constraint required appears to be something like an order constraint, as Heim suggested in the quote above. Schlenker developed a very general way of making accounts of presupposition projection sensitive to order in the way the Karttunen/Heim projection rules are. ${ }^{24}$ Schlenker (2006, 2008a, 2009) builds in the order component by using a kind of incremental checking routine: one has to check at each stage of the derivation that all presuppositional expressions mentioned so far will be satisfied no matter what formulas follow.

Although Schlenker's incremental checking routine could be put on top of the loose system above, ${ }^{25}$ a simpler way to replicate the Heim/Karttunen rules is to build an order constraint into the notion of rewrite rules used. An order-sensitive rewrite rule for the formula $\alpha[\psi * \phi]$ is a rewrite rule that does not allow any instance of the CCP $\psi$ to operate on a formula that contains $\phi$. Corresponding to this more constrained notion of a rewrite rule is a refinement of our previous semantics for complex CCPs:

24 The order constraint may operate on the purely linear order of a sentence, but it is more likely that it works on some less superficial kind of order (i.e. order at a syntactic or semantic level of representation). For example, it is widely thought that conditionals have the same presupposition projection whether the antecedent appears before or after the consequent (see, e.g., Heim 1990):

(26) a. John will know that there's been a break-in, if there has been one.

b. If there has been a break-in, John will know it.

25 This is what I did in the earlier versions of this system (Rothschild 2008b). 
Daniel Rothschild

(27) $\alpha[\phi * \psi]$ is defined iff a) there exists an order-sensitive, truthconditionally adequate rewrite rule for $\alpha[\phi * \psi]$ whose semantic value is defined and $b$ ) all such rewrite rules have the same semantic value. When it is defined $\alpha[\phi * \psi]$ has that unique semantic value.

It takes little effort to show that on this system we reproduce Karttunen/Heim projection rules, as the following proposition shows.

Proposition 2. Suppose $\phi$ and $\psi$ are CCPs with monotonic definedness conditions and intersective meanings and $\alpha$ is a sentence. It follows on the semantics of (27) that the projection properties match those of Heim's system, listed here:

- $\alpha[\neg \phi]$ is defined iff $\alpha \backslash \alpha[\phi]$ is defined.

- $\alpha[\phi \wedge \psi]$ is defined iff $(\alpha[\phi])[\psi]$ is defined.

- $\alpha[\phi \vee \psi]$ is defined iff $(\alpha[\neg \phi])[\psi]$ is defined.

- $\alpha[\phi \rightarrow \psi]$ is defined iff $(\alpha[\phi])[\psi]$ is defined.

\section{Individual connectives: Theory and data}

The preceding discussion has been rather abstract. In this section, I will review the predictions the two semantics in the previous section make for different connectives and discuss how they compare both to other accounts and to what we find empirically.

\subsection{And}

Background Conjunction is somewhat special as there is a pragmatic story of presupposition projection in conjunctions, due to Stalnaker (1974). Stalnaker's trick is to view conjunctions, pragmatically, as consecutive assertions. If all goes well, by the time we get to the second assertion (i.e. the second conjunct), the common ground has already been updated with the first assertion. In this case we should expect the Karttunen/Heim rule for conjunction. ${ }^{26}$ The most basic problem that this pragmatic story faces is that it does not naturally extend to embedded uses of conjunction. For example, a conjunction

26 Schlenker (2008a, 2009, 2010) has extensively criticized this account and all points I make here can be found in his work. 
Explaining presupposition projection

inside the antecedent of a conditional yields the same presupposition as a conjunction outside of one. In the case of an embedded conjunction, neither conjunct is asserted so the consecutive assertion account of conjunction cannot apply.

The only way to deal with such cases and maintain the pragmatic story, and what I take Stalnaker (2010) to be doing in his response to Schlenker, is to view the antecedents of conditionals as being suppositionally asserted, and thus view the conjunction here as a set of two suppositional assertions. The presupposition triggers, then, respond to suppositional common grounds rather than real common grounds in conditionals. ${ }^{27}$ For this theory to be genuinely predictive (for embedded conjunctions) we need an account of all the types of common ground relevant in any arbitrary embedded context. I do not know of any such account, so I will put aside this suggestion here and conclude that the pragmatic strategy is, if not unworkable, at least unworkedout. Pragmatic theories do not generalize compositionally, as Karttunen's and Heim's theory do.

Explanatory dynamic account On the symmetric version of my account, any adequate rewrite rule is acceptable for conjunction. As in all cases, there are only two relevant rewrite rules for $\alpha[\phi \wedge \psi]:(\alpha[\phi])[\psi]$ and $(\alpha[\psi])[\phi]$. The reason these are the only options worth considering is that one of these is defined iff some other rewrite rule is defined. Thus, the loose rewrite semantics, without the order constraints, predicts that conjunction can allow filtering of presuppositions in either direction. If we add the order constraint, then the loosest rule we can use is $(\alpha[\phi])[\psi]$, so we reproduce the Karttunen/Heim projection rule.

It is not easy to find empirical evidence about which version is better. To get clear examples we first have to rule out the possibility that the relevant judgments are due to violating pragmatic maxims against redundancy (Schlenker 2008a). One way to do this is to negate the presuppositional expression and so consider pairs like this:

(28) a. Mary is pregnant, and John doesn't know it.

b. John doesn't know Mary is pregnant, and she is.

It seems me that (28a) is distinctly better than (28b). However, if we replace and with but in (28b), then the situation is less clear. The judgments here are

27 This style of explanation also seems to me what Soames (1982) has in mind. 
subtle and controversial enough that introspective judgments are not going to decide this question. ${ }^{28}$

\section{$6.2 \mathrm{Or}$}

Background The treatment of the projection properties of or has attracted much less of a consensus then that of and. Indeed, Heim's original paper does not even given a dynamic rule for $o r$. Proposed rewrite rules vary from rules that do not allow any presupposition filtering such as $\alpha[\phi \vee \psi]=$ $\alpha[\phi] \vee \alpha[\psi]$ to the rule I used here that allows filtering of the second presupposition by the negation of the first: $\alpha[\phi \vee \psi]=\alpha \backslash((\alpha[\neg \phi])[\psi])$.

What is interesting about disjunction, and most difficult for traditional dynamic theories, is that no rule for disjunction seems to capture the empirical facts about presupposition projection in disjunction. It has long been recognized that disjunction appears to have symmetric properties of presupposition projection. So the following two examples seem equally acceptable when there is no presupposition that John used to smoke: ${ }^{29}$

(29) a. Either John didn't use to smoke or he stopped.

b. Either John stopped smoking, or he didn't use to.

What has not been observed to my knowledge is that there is no adequate rewrite rule that yields symmetric predictions for disjunction. ${ }^{30}$ Thus, disjunction alone provides a strong empirical argument against the stipulation of unique rewrite rules for each connective in dynamic semantics. This, then, suggests that if we are going to have a dynamic system, we should have a symmetric semantics such as the one I propose here.

Other explanatory approaches in the literature give symmetric projection rules of disjunction that can capture the acceptability of both examples in (29). Not all of them do so in satisfactory ways, though. As I pointed out in my reply to Schlenker (2008a), the predictions of Schlenker's system while they do capture both examples in (29) are, in fact, extremely liberal and

28 Chemla \& Schlenker (2009) provide empirical results on this question - and for symmetric readings of other connectives - providing limited evidence for the symmetric readings being somewhat acceptable.

29 We can replace used to smoke with used to smoke heavily in both examples to eliminate the possibility of a Gazdar-style explanation of this symmetry.

30 The reason this is true that for any single adequate rewrite rule for $\alpha[\phi \vee \psi]$ to be defined either $\alpha[\phi]$ needs to be defined or $\alpha[\psi]$ needs to be defined. 
Explaining presupposition projection

so also predict that presuppositions can cancel each other across disjunction. ${ }^{31}$ So, the following example would be acceptable on his system with no presupposition:

(30) Either he doesn't regret that he used to smoke, or he didn't stop smoking.

This seems to me like a bad prediction. By contrast, the trivalent accounts of presupposition projection (George 2007, Fox 2008) give symmetric readings without this problem.

Explanatory dynamic account For disjunction the two relevant rewrite rules are $\alpha[\phi \vee \psi]=\alpha \backslash(\alpha[\neg \phi])[\neg \psi]$ and $\alpha[\phi \vee \psi]=\alpha \backslash(\alpha[\neg \psi])[\neg \phi]$. Again, we can restrict our consideration to these two rules since if any adequate rewrite rule is defined, one of these two rules will be as well. The availability of both these rules gives us the symmetric definedness conditions for conjunction. We do not, however, over-generate and allow presuppositions to cancel each other in examples like (30).

When we add in the order constraint the only relevant rule possible is $\alpha[\phi \vee \psi]=\alpha \backslash(\alpha[\neg \phi])[\neg \psi]$ which only allows filtering of presuppositions in the second disjunction by the negation of the first disjunct.

\subsection{If}

Background It is widely accepted that the material conditional account of if is inadequate for natural language conditionals. ${ }^{32}$ Nonetheless, following Heim (1983b) and Schlenker (2008a), I give a semantic analysis of conditionals that is equivalent to the material conditional account. This is of some use, as the material conditional is, in many instances, truth-conditionally equivalent to more sophisticated analyses.

With conditionals the most standard generalization, and that given in the Heim/Karttunen rules above, is that the presupposition of the antecedent is projected out of the clause, but that the presupposition of the consequent is only projected to the extent that it is not entailed by the antecedent. So,

31 See Rothschild 2008b. Beaver (2008) makes a similar point in his reply in the same volume. A similar point can also be made about other binary connectives, but it is most relevant for disjunction as this is a clear case where we need a symmetric rule. Schlenker (2008c) suggests a repair strategy to deal with this problem but gives little motivation for it. 32 Some of the many arguments are reviewed in Kratzer 1986 and Edgington 1995. 
the presupposition of a sentence of the form $A \rightarrow B$ is the same as that of a sentence of the form $A \wedge B$. This is captured by, e.g., the rewrite rule $\alpha[\phi \rightarrow \psi]=\alpha \backslash((\alpha[\phi])[\neg \psi])$.

Explanatory dynamic account On the loose rewrite semantics, the two relevant rewrite rules for the material conditional are $\alpha[\phi \rightarrow \psi]=\alpha \backslash(\alpha[\phi])[\neg \phi]$, and $\alpha[\phi \rightarrow \psi]=\alpha \backslash(\alpha[\neg \psi])[\phi]$. Once we add the order constraint the only rule that is applicable is the first, which gives the standard predictions.

But what about the reverse rule, $\alpha[\phi \rightarrow \psi]=\alpha \backslash((\alpha[\neg \psi])[\phi])$ ? It is hard to find any clear evidence that this rule has a role to play. Testing it requires evaluating a sentence where the presupposition of the antecedent is satisfied by the negation of the consequent. Some simple examples of this kind can be ruled out on the ground of having some other pragmatic infelicity:

(31) If John knows Mary is pregnant, then she isn't. (Conditional makes no sense).

If we complicate this by adding negation we can get a clearer example:

(32) If John doesn't know that Mary is pregnant, then she isn't.

This sentence is acceptable without a presupposition, but it is not clear if this is because of a sort of backwards filtering allowed by the reverse rule, or just plain cancellation. After all, it is possible to say:

(33) John doesn't know that Mary is pregnant, because she isn't.

So we would need more complex, controlled examples to test the possibility (or lack thereof) of reverse filtering in conditionals. I cannot find any cases where I have clear judgments, so again, it is not clear how to decide between the symmetric and the asymmetric versions of the theory.

\subsection{Other connectives}

Background We criticized Heim's account for failing to make predictions about the presupposition projection properties of arbitrary truth-functional connectives. Schlenker (2008a) emphasizes that with respect to a connective like unless we would hope that a theory of presupposition projection would be able to tell us, given its truth-conditions, what its projection properties will be. More generally, we should expect that any two connectives with the same 
Explaining presupposition projection

truth-conditional properties should have the same projection properties. A natural case of this is found with and and but, which according to most are truth-conditionally equivalent, with but being distinct in virtue of having additional, non-truth-conditional force.

Explanatory dynamic account If we are to treat unless as a connective with a truth table (which, as with conditionals, requires a simplification of its semantics) then the natural truth conditions are $\alpha$ unless $\beta$ iff $\alpha \vee \beta$. In this case we should expect it to have the projection properties of disjunction. This seems to make good predictions as we often get disjunction-like patterns of presupposition projection. For instance, the negation of the first part of a sentence connected with unless can satisfy a presupposition in the second part:

(34) Unless he didn't talking to her yesterday, John will regret talking to Mary.

As with conditionals, it is difficult to tell whether the symmetric predictions from the looser systems are also found.

With but it seems clear that the projection properties are exactly those found with and, a prediction that this explanatory version of dynamic semantics makes.

(35) John is sick, but Bill doesn't know it.

One final note about other connectives: I will not attempt a proof here, but I am confident that across arbitrary truth-table-definable propositional connectives, the order-sensitive account is equivalent to the order-sensitive predictive accounts proposed by Schlenker (2008a, 2009), Chemla (2008), Fox (2008), and George (2007). ${ }^{33}$

\section{Adding quantification}

Heim's paper is also well known for its systematic treatment of presupposition projection under quantifiers. In essence, adding quantification does nothing to change the conclusions that we made for binary quantifiers. As with binary connectives, the semantics that Heim gives would require stipulations for each quantifier in order to capture the presupposition projection

33 See the appendix of Schlenker 2008b and Rothschild 2008a for discussion of the equivalence of predictions across different explanatory accounts. 
facts (or rather, what she takes to be the facts). If we extend the loose semantics above to include quantifiers in a natural way, then we can capture, without stipulations, these same generalizations.

Expressions that trigger presuppositions can have variables in them. So, for instance, sentences of the form $x$ stopped smoking give rise to the presupposition that $x$ used to smoke. We can then bind such variables with quantifiers in examples like this:

(36) Every student stopped smoking.

$=$ Every $_{x}$ (student $x$, stopped smoking $x$ )

Heim discusses sentences of this form and gives general predictions for how they project presuppositions. She argues that the presupposition of a sentence of the form $\operatorname{Every}_{x}(f x, g x)$, where $g x$ presupposes that $x$ satisfies $\underline{g}$ and $f x$ has no presupposition, is that every object satisfying $f$ satisfies $\underline{g}$. Returning to (36) we can state Heim's prediction as follows:

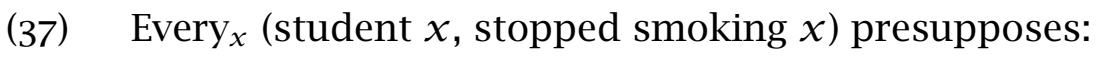

Every $_{x}$ (student $x$, used to smoke $x$ )

To summarize: the presuppositions in the matrix predicate of a universal quantifier are universal across all objects satisfying the restrictor predicate.

Heim goes beyond every and argues that the dynamic semantic framework will make predictions of the same form for all quantifiers. As with binary connectives, this claim turns out to be overly optimistic (even if we restrict ourselves to rewrite semantics for quantifiers): in fact, separate stipulations are needed for each quantifier. In the rest of this section, I will show that, if we assume that quantifiers are conservative and both predicates are related to the truth conditions in a non-trivial way, we can replicate Heim's generalizations with the semantics in \$5.1-5.2, suitably expanded to include quantification. Some may wish to skip the rest of this section, where I demonstrate these points, as the technical details are rather involved without containing much of interest beyond what was already in the propositional case. In the next section, I will discuss the empirical adequacy of the rules given here in a more informal way.

\subsection{Syntax}

- lower-case letters $a, b, c \ldots$ are atomic sentences

- upper-case letters $F, G, H \ldots$ are $n$-place CCPs predicates 
Explaining presupposition projection

- $x_{1}, x_{2} \ldots$ are variables

- Every, Some, Most... are quantifiers (we use $Q$, below, to represent an arbitrary quantifier)

- the set of CCPs is defined as follows:

- if $F$ is an $n$-place CCP predicate, and $x_{1} \ldots x_{n}$ are variables, then $F\left(x_{1} \ldots x_{n}\right)$ is a CCP

- if $\phi$ and $\psi$ are CCPs and $x_{i}$ is a variable, then $\phi \wedge \psi, \neg \phi, \phi \vee \psi$, $\phi \rightarrow \psi$, and $Q_{i}(\phi, \psi)$ are CCPs

- the set of complex sentences is defined as follows:

- any atomic sentence is a sentence

- if $\alpha$ and $\beta$ are sentences and $x_{i}$ is a variable, then $\alpha \wedge \beta, \alpha \vee \beta$, $\alpha \backslash \beta$ and $Q_{i}(\alpha, \beta)$ are sentences

- if $\alpha$ is a sentence and $\phi$ is a CCP, then $\alpha[\phi]$ is a sentence

\subsection{Partial semantics with quantifiers and variables}

The main difference in the semantics from the propositional case is that all sentences now denote sets of world/assignment-function pairs rather than just sets of worlds. A set of world/assignment-function pairs is a subset of $\{(f, w): f$ is an assignment function and $w$ is a world $\}$, where assignment functions are functions from variables to individuals. Intuitively, sentences denote the set of all those pairs on which the sentence is true.

The semantic value of a sentence is defined relative to an interpretation, $I$. For every $n$-place atomic CCP predicate $F, I$ assigns $F$ two different $n$-place relations (across possible worlds), the first we will call the presuppositional relation and the second the assertive relation. We can model these relations by functions from worlds to $n$-tuples of individuals. ${ }^{34}$

For any atomic CCP, $F\left(x_{1} \ldots x_{n}\right)$, and any sentence, $\alpha, \llbracket \alpha\left[F\left(x_{1} \ldots x_{n}\right)\right] \rrbracket$ is defined iff for every pair $(f, w)$ in $\llbracket \alpha \rrbracket,\left(f\left(x_{1}\right) \ldots f\left(x_{n}\right)\right)$ is in the extension of the presuppositional relation associated with $F$ at $w$. If $\llbracket \alpha\left[F\left(x_{1} \ldots x_{n}\right)\right] \rrbracket$

34 Formally, then, $I$ consists of is a tuple $(W, D, S, C)$, where $W$ is a set of possible worlds, $D$ is a set of individuals (which we think of as constant across worlds), $S$ is a function from atomic sentences to sets of world/assignment-function pairs, $C$ is a function from atomic CCPs to an ordered pair of relations. Assignment functions go from variables to elements of $D$. And relations are functions from $W$ to $P(D)$. 
is defined then its semantic value is $\left\{(f, w) \in \alpha:\left(f\left(x_{1}\right) \ldots f\left(x_{n}\right)\right)\right.$ is in the assertive relation of $F$ at $w$ \}.

The recursive semantic rules for the connectives (outside of CCPs) $\wedge, \vee$, and $\backslash$ are the same as the propositional case, which are listed in (7). We need to treat quantifiers operating on sentences. To do this we associate with each quantifier $Q$ a binary relation $R_{Q}$, as is standard in the theory of generalized quantifiers. Our semantics for quantifiers is then stated as follows:

$$
\left.\left.\llbracket \mathbf{Q}_{i}(\alpha, \beta) \rrbracket=\left\{(f, w):\left\{o:\left(f_{x_{i} \rightarrow o}, w\right) \in \alpha\right)\right\} R_{Q}\left\{o:\left(f_{x_{i} \rightarrow o}, w\right) \in \beta\right)\right\}\right\}
$$

This will only work (intuitively) if $x_{i}$ is free in $\alpha$ and $\beta$. By definition, $x_{i}$ is free in $\alpha$ iff for all $w$ and $f^{\prime}$ that differ from $f$ only in their assignment to $x_{i}$, if $(f, w)$ is in $\alpha$, then so is $\left(f^{\prime}, w\right)$. For an example of how the semantics for quantifiers works, consider $M=\left\{(f, w): f\left(x_{i}\right)\right.$ is a man in $w\}, T=\left\{(f, w): f\left(x_{i}\right)\right.$ is tall in $\left.w\right\}, R_{Q}=\subset$. Then $Q_{i}(M, T)=\{(f, w)$ : the set of all men in $w$ is a subset of the the set of all tall things in $w$ \}. So, in this case, $Q$ is the universal quantifier, every.

\subsection{Heim's semantics for complex CCPs}

Heim's rules for the standard connectives and negation are as they were described in \$3. The only addition needed is the treatment of quantifiers within CCPs. Her rewrite rule for every can be stated as follows:

$$
\alpha\left[\operatorname{Every}_{i}(\phi, \psi)\right]=\alpha \wedge \operatorname{Every}_{i}(\alpha[\phi],(\alpha[\phi])[\psi])
$$

For this rule to work we need to assume that $x_{i}$ is free in $\alpha$. If we look at (39) we can see that $\alpha\left[\right.$ Every $\left._{i}\left(F x_{i}, G x_{i}\right)\right]$ is defined iff $\alpha\left[F\left(x_{i}\right)\right]$ and $\left(\alpha\left[F x_{i}\right]\right)\left[G x_{i}\right]$ are both defined. Supposing $x_{i}$ is free in $\alpha$, the definedness condition is that for every world $w$ that appears in the denotation of $\alpha$ every individual in $w$ must satisfy the presupposition of $F$ and every individual which satisfies $F$ must also satisfy the presupposition of $G$ for the formula to be defined.

As with binary connectives, Heim could have defined things differently and gotten the same basic truth conditions. Indeed, the simplest definition is as follows:

(40) $\alpha\left[\operatorname{Every}_{i}(\phi, \psi)\right]=\alpha \wedge \operatorname{Every}_{i}(\alpha[\phi], \alpha[\psi])$

This would have $\alpha\left[\right.$ Every $_{i}\left(F\left(x_{i}\right), G\left(x_{i}\right)\right]$ presuppose that for every world $w$ that appears the denotation of $\alpha$ every individual in $w$ must satisfy the 
Explaining presupposition projection

presupposition of $F$ and the presupposition of $G$. So this would give us much stronger presuppositions than Heim actually predicts.

In order to get general predictions of presupposition projection for each quantifiers, Heim needs to stipulate in the definition for all quantifiers a form like that of (39) to ensure that the quantifier triggers the right presuppositions. So, we might want, again, to find a system that can produce these results without resort to such stipulations.

It is worth nothing in this context how facts about anaphora fit into the picture here. In Heim 1982, 1983a the semantics for every in (39) is motivated by consideration of anaphora. In particular, Heim wanted to ensure that an indefinite description in the restrictor of a quantifier could be anaphorically picked out by a definite pronoun in the matrix. ${ }^{35}$ Such anaphora, in the context of Heim's treatment of pronouns and indefinites (both of which are variables), would be allowed by (39) but not by (40). It may seem then, that in a language with variables, anaphora provides an independent motivation for Heim's semantics. It seems to me, however, that this is not the right way to think about things. Rather we should, like Heim, view anaphora and presupposition as two related problems which require a common solution. Thus, that the semantics for every in (39) provides a natural treatment of anaphora in quantification expressions, is not an independent consideration in favor of that semantics. Anaphora does not solve the explanatory problem.

\subsection{Loose semantics with order constraint}

What we need is a general definition of the meaning of complex CCPs that can include quantifiers. We will maintain our previous definitions for CCPs whose top operator is a binary connective. But we need a new rule for CCPs whose top operator is a quantifier. To do this we will define again two concepts: a) being a rewrite rule for $\alpha\left[Q_{i}(\phi, \psi)\right]$ and b) the property of a rewrite rule being truth-conditionally adequate.

The recursive definition of a rewrite rule is the same as it was in the propositional case, given in (13), except we also allow adding quantifiers to connect two sentences. The definition of truth-conditional adequacy is the same as propositional case, given in (16), except we need to use a classical logic with variables and generalized quantifiers rather than a propositional logic to test the adequacy of rewrite rules with quantification. Using these

35 A similar argument could be made with respect to Heim's semantics for conjunctions and conditionals. 
definitions for rewrite rules and adequacy, we can, again, consider a system in which a complex CCP is defined if and only if there is a truth conditionally adequate, order-sensitive, rewrite rule defined for it (order sensitivity is defined as before) as in (27). ${ }^{36}$

The resulting semantics makes different predictions for different logically possible quantifiers. However, luckily, it makes the same predictions for about the presupposition projection properties of all quantifiers that have two features shared by all natural language quantifiers. Here are the two features:

Conservativity A quantifier $Q_{i}$ is conservative if and only if for any atomic formulas $a, b: Q_{i}(a, b)$ is logically equivalent to $Q_{i}(a, b \wedge a)$.

Non-Triviality of Matrix and Restrictor The matrix and restrictor predicates are non-trivial iff there is no formula logically equivalent to $Q_{i}(a, b)$ that does not quantify over both $a$ and $b$.

For quantifiers with these two properties we can now state the result giving us Heim's universal projection: ${ }^{37}$

Proposition 3. For a quantifier $Q$ satisfying both the Conservativity and NonTriviality, CCPs $\phi$ and $\psi$, variable $x$, and sentence $\alpha, \alpha\left[Q_{i}(\phi, \psi)\right]$ is defined iff $\alpha[\phi]$ is defined and $(\alpha[\phi])[\psi]$ is defined.

This result show that the order-sensitive semantics with quantification reproduces Heim's projection rules for quantifiers: the presupposition of the matrix predicate is only projected for those individuals that satisfy the restrictor.

\section{Quantifiers: Theory and data}

\subsection{Existential quantifiers}

As this loose system shares Heim's (1983b) predictions, it also faces the same serious empirical challenges. A major one, which Heim discusses, is the

36 I am limiting the discussion here to the order-sensitive rule. However, it may be that the non-order-sensitive version is also of interest.

37 Note that our partial semantics builds monotonic definedness conditions and intersective meanings into the definitions of atomic CCPs, so we need not state these separately to derive the result. 
Explaining presupposition projection

universal presuppositions predicted under existential quantifiers. Consider, for instance:

(41) A man stopped playing the guitar.

If we think of a man as a regular existential quantifier, then we presuppose that every man was playing the guitar earlier. Heim discusses examples of this form and argues that the predicted presupposition is too strong.

Her somewhat notorious solution is to posit a process of "local accommodation" by which the presupposition is only accommodated for what, in effect, is an existential witness (or the intended referent) of the indefinite in (41) rather than the entire domain of men. In our loose semantics we could also help ourselves to local accommodation to deal with this problem. ${ }^{38}$ However, using local accommodation as the explanation for the systematically weak presuppositions found in (41) effectively begs the question of why existential quantifiers in particular give rise to weak presuppositions, since local accommodation is a technical possibility for any quantifier.

There are two salient alternative strategies. The first is to use domain restrictions to explain the limited presuppositions of examples like (41). The second is to rework the semantics of existential quantifiers (and possibly other quantifiers as well) in a way that yields systematically weaker presuppositions. I will briefly discuss both of these options.

Schlenker (2008a) proposes to explain the weak presuppositions of sentences like (41) by appeal to a covert domain restriction. ${ }^{39}$ The sort of domain restriction needed to explain the particularly weak presupposition of (41) would be the one proposed by Schwarzschild (2002) in his theory of "singleton indefinites". Schwarzschild argued that the domain restriction of an indefinite quantifier could be as narrow as a single object. If such a domain restriction were in the syntax of (41) as part of the restrictor, then we would predict that the presupposition in the restrictor would only project onto the one individual in the domain.

Is the use of domain restrictions any less stipulative than simply allowing local accommodation? Technically, one could use domain restrictions to

38 Heim's (1983b) system may seems superficially very different here, as she does not treat $a$ man as a quantifier but rather as just a free variable. However, since it is the assumption that the variable is free that is doing all the work, the system is not greatly different from the approach here couched in terms of generalized quantifiers.

39 See von Fintel 1994, Gawron 1996, Geurts \& van der Sandt 1999 for general discussion of domain restriction in semantic theory. 
reduce the presuppositions under universal quantifiers as well as existential ones. For example, in this sentence:

Every man played his guitar.

If we restrict the domain to one individual, then we would generate a very weak presupposition for (42). However, we would also do violence to the meaning as the sentence would now only be about that one individual. With existential quantifiers, as Schwarzschild pointed out, narrow domain restrictions have little effect on meaning. Domain restriction, thus, has the advantage over local accommodation of providing for a principled distinction between the presupposition projection behavior of different quantifiers. ${ }^{40}$

A more radical option for explaining the systematically weak presuppositions of existential quantifiers is to rethink the semantic treatment of variables and quantification. Beaver (1992, 1994, 2001) explores different variations on Heim's dynamic semantics that allow for quantifiers, particularly existential ones such as indefinite descriptions, to yield weaker presuppositions. Some of Beaver's semantic tools could be adopted in the dynamic system presented here in one of two fashions: either systematically in the definition of rewrite rules, thereby yielding weaker presuppositions for all quantificational expressions, or exceptionally for certain existential quantifiers, yielding weak presuppositions for those quantifiers alone. There are problems with either approach. Heim's prediction of universal projection was motivated by intuitions about the presupposition projection behavior of no and every, both of which seem to lead systematically to universal pre-

40 David Beaver (p.c.) raised a basic challenge for the domain-restriction approach: it seems like indefinites with singleton domains cannot get intuitively narrow-scope readings under negation. If this is right, then domain restrictions will not be able to explain the possibility of existential presupposition in indefinites that scope under negation, as in this example:

(43) I've been on the lookout for years, but I've never seen a man playing his guitar like he really cared.

There are at least two possible responses to this worry: First, a non-singleton domain restriction might be able to deal with this example, e.g. a domain restriction to the set of male guitar players. Such a restriction would still give the sentence its intuitive reading while not resulting in the strong presupposition that all men own guitars. Second, it is arguably possible for singleton domains to nonetheless give rise to narrow scope readings if the singleton restriction is chosen carefully (Breheny 2003, Rothschild 2007).

A related worry for the domain-restriction explanation of weak presuppositions is that it predicts that the capacity for extraordinary wide scope is co-extensive with the capacity for existential presuppositions and that prediction does not seem quite right (Schlenker, p.c.). 
Explaining presupposition projection

suppositions, as in (42). ${ }^{41}$ If we adopt a weaker semantics across the board we will lose these predictions. On the other hand, if we only adopt Beaver's alternative semantics for indefinites and other existential quantifiers, we sacrifice our explanatory ambition.

My purpose here is just to indicate that the same theoretical options for explaining the presuppositions triggered under existential quantifiers are available to the explanatory dynamic account presented here as are available to other versions of dynamic semantics. I leave it as open question whether an empirically adequate, explanatory account is possible. ${ }^{42}$

\subsection{Restrictors}

It is worth noting that a related issue arises with regard to presuppositions triggered in the restrictors of quantifiers, a topic less commonly discussed in the presupposition literature. ${ }^{43}$ Any presupposition in the restrictor of a quantifier is predicted to project universally across the domain on Heim's semantics as well as the explanatory dynamic account here. These predictions might seem much stronger than what we actually observe. Common nouns seem to trigger sortal presuppositions of various sorts. So, for example, bachelor might presuppose male and marriageable: after all, saying $x$ is not a bachelor, seems to take for granted that $x$ is male and not a Catholic priest. If this is correct, then on the theory given here a sentence like (44) presupposes that every element in the domain is marriageable. ${ }^{44}$

(44) Every bachelor is happy.

This is an unacceptably strong prediction, though it might be made more palatable if we limit our attention to the restricted domain presupposed for any given use of (44). More serious problems arise when we consider

41 See Chemla 2009 for some interesting experimental data supporting this claim. However, Chemla's data also indicates that Heim's predictions are not successful for many other quantifiers.

42 If we put aside indefinite quantifiers like $a$ man we may be able to give a robust defense of Heim's predictions for all other quantifiers, including existential quantifiers such as some man: Charlow (2009) argues that when we use strong presuppositions which have been argued to be incapable of being accommodated, such as too, only universal presuppositions are possible.

43 Some relevant discussions are Beaver 2001, Schlenker 2008a and Chemla 2009.

44 Emmanuel Chemla suggested this type of case to me. 
presuppositions triggered in a relative clause in the restrictor of a quantifier:

(45) Every student who knows he failed the exam will want to leave the room before the results are announced.

Here the presupposition trigger knows he failed the exam applies after the common noun student, so Heim's theory and our theory predict that (45) presupposes that every student failed the exam. Even if we restrict attention to the students who took the exam - what might seem like the most natural domain - this seems like a strong presupposition as it is incompatible with any student passing the exam.

The situation is very similar to the one we discussed above with existential quantifiers. Local accommodation might be appealed to, but it does not have the capacity to explain the apparent difference in projection between presuppositions in restrictors and presuppositions in matrices, a difference which Chemla's (2009) empirical study supports. Again, we have two basic alternatives to local accommodation: positing strong domain restrictions that limit the presuppositions ${ }^{45}$ and giving a different semantic system that systematically predicts weaker presuppositions. Both of these options, like local accommodation, will give us a mechanism for limiting the strength of presuppositions triggered in the restrictors of quantifiers, though I am not sure how satisfying either is.

It should be clear from these two brief discussions that the theoretical and empirical challenges raised by presupposition projection under quantification are considerable. I have tried to point out some of the problems facing the account here and some ways of responding to them, but whether a satisfactory account can be developed along these lines is very much an open question.

\section{Conclusion}

I have shown that Heim's treatment of presupposition projection can be extended to generate the same results without the stipulations. However, the loose semantics introduced here may seem to some to be closer in spirit to a trivalent semantic system, like the strong-Kleene truth tables, than it does to Heim's original semantics. A more sustained defense of dynamic semantics

45 Using domain restrictions to limit presuppositions in this context bears a strong resemblance to the process of "intermediate accommodation" in DRT (van der Sandt 1992, Geurts \& van der Sandt 1999). 
Explaining presupposition projection

would need to show that the extra complexity of the system (the treatment of sentences as expressing CCPs rather than partial propositions) is doing real work for us.

\section{Appendix: Proofs}

Proposition 1. Suppose $\phi$ and $\psi$ are CCPs with monotonic definedness conditions and intersective meanings and $\alpha$ is a sentence, it follows on the semantics of (2O) that:

- $\alpha[\neg \phi]$ is defined iff $\alpha[\phi]$ is defined.

- $\alpha[\phi \wedge \psi]$ is defined iff $(\alpha[\phi])[\psi]$ or $(\alpha[\psi])[\phi]$ is defined.

- $\alpha[\phi \vee \psi]$ is defined iff $(\alpha[\neg \phi])[\psi]$ or $(\alpha[\neg \psi])[\phi]$ is defined.

- $\alpha[\phi \rightarrow \psi]$ is defined iff $(\alpha[\phi])[\psi]$ or $(\alpha[\neg \psi])[\phi]$ is defined.

Proof. In each case, the right-to-left direction is easier than the left-to-right direction. For the right-to-left direction, all we need to show is that there is a truth-conditionally adequate rewrite rule for the relevant connective that is defined whenever the condition on the right is met. (Given the intersective meaning assumption and the definition of truth-conditional adequacy, if two rewrite rules are defined they will always yield the equivalent meaning.) Here we state truth-conditionally adequate rewrite rules that are defined whenever the condition on the right is met:

Negation $\alpha \backslash \alpha[\phi]$ will be defined.

Conjunction Either $(\alpha[\phi]) \psi$ or $(\alpha[\psi]) \phi$ will be defined.

Disjunction Either $\alpha \backslash((\alpha[\neg \phi])[\neg \psi])$ or $\alpha \backslash((\alpha[\neg \psi])[\neg \phi])$ will be defined. ${ }^{46}$

Conditional Either $c \backslash((\alpha[\phi])[\neg \psi])$ or $c \backslash((\alpha[\neg \psi])[\phi])$ will be defined.

What remains, then, is the left-to-right direction. This requires proving for each complex CCP, if the complex CCP on the left is defined according to (20), then the condition on the right is satisfied. Given the semantics of (20), this is equivalent to showing that if the right-hand side condition is not met, then there is no truth-conditionally adequate rewrite rule for the expression

46 I use $\alpha[\neg \phi]$ as a shorthand for $\alpha \backslash \alpha[\phi]$. This saves a lot of space, and, as we are proving, they are equivalent in both definedness and denotation when defined. 
on the left-hand-side that is defined. For each connective, we will prove by induction on the complexity of rewrite rules, as defined in (13), that if the condition on the right-hand side is not defined then no defined rewrite rules for the connective exists. I work it out in detail for the case of conjunction and sketch the proofs for the other cases (all of which are quite similar).

Conjunction Suppose neither $(\alpha[\phi]) \psi$ nor $(\alpha[\psi]) \phi$ is defined. Let's suppose now that $\alpha[\phi]$ is defined but $\alpha[\psi]$ is not (they cannot both be defined on the supposition of the previous sentence, given the monotonic definedness conditions). On these assumptions we will show there is no truth-conditionally adequate rewrite rule for $\alpha[\phi \wedge \psi]$ that is defined. To do this, we will show by induction on complexity that every defined rewrite rule is either entailed by $\alpha[\phi]$ or entails $\neg \alpha[\phi]$. (Here we use the $\neg$ sign applying to sentences in the usual sense: $\neg \alpha$ denotes the complement of $\alpha$ ) If this holds, then no defined rewrite rule will be truth-conditionally for conjunction, since no rewrite rule that is truth-conditionally adequate for $\alpha[\phi \wedge \psi]$ can have either of these logical entailment properties.

Base step: only formula is $\alpha$, so trivial. Induction step: Suppose $\gamma$ and $\gamma^{\prime}$ are rewrite rules for $\alpha[\phi \wedge \psi]$ satisfy the inductive property of either begin logically weaker than $\alpha[\phi]$ or stronger than $\neg \alpha[\phi]$. (Note: "weaker than" and "stronger than" are used in non-strict sense throughout.) We have two ways of getting more complex rewrite rules for $\alpha[\phi \wedge \psi]$ : adding a CCP to $\gamma$ or $\gamma^{\prime}$ or using the connectives $\backslash, \wedge$, and $\vee$ to connect $\gamma$ and $\gamma^{\prime} .{ }^{47}$ I will go through these in turn:

Adding $\phi$ : Suppose $\gamma$ is logically weaker than $\alpha[\phi]$. Then adding $[\phi]$ will not change this property. Suppose $\gamma$ is logically stronger than $\neg \alpha[\phi]$. Then, adding [ $\phi]$, given its intersective meaning, will not change this property.

Adding $\psi$ : Suppose $\gamma$ is logically weaker than $\alpha[\phi] . \gamma[\psi]$, if it were defined might not have the property, but it will not be defined since 1) $(\alpha[\phi])[\psi]$ is not defined 2) $\gamma$ is weaker than $\alpha[\phi]$ by assumption and 3) $\psi$ has monotonic definedness conditions. If $\gamma$ is stronger than $\neg \alpha[\phi]$, then $\gamma[\psi]$ will be as well.

Forming $\gamma \wedge \gamma^{\prime}$ Suppose $\gamma$ and $\gamma^{\prime}$ are weaker than $\alpha[\phi]$. Then so is their conjunction. And if one of the two is stronger than $\neg \alpha[\phi]$,

47 This can be seen by the recursive definition of rewrite rules in (13). 
Explaining presupposition projection

then their conjunction is too.

Forming $\gamma \vee \gamma^{\prime}$ Suppose $\gamma$ and $\gamma^{\prime}$ are both stronger than $\neg \alpha[\phi]$. Then, so is their disjunction. And if one of the two is weaker than $\alpha[\phi]$ then their disjunction is too.

Forming $\gamma \backslash \gamma^{\prime}$ If $\gamma$ is stronger than $\neg \alpha[\phi]$ then so is $\gamma \backslash \gamma^{\prime}$. If $\gamma^{\prime}$ is weaker than $\alpha[\phi]$ then $\gamma \backslash \gamma^{\prime}$ is stronger than $\neg \alpha[\phi]$. This leaves the case in which $\gamma$ is weaker than $\alpha[\phi]$ and $\gamma^{\prime}$ is stronger than $\neg \alpha[\phi]$. In this case, $\gamma \backslash \gamma^{\prime}$ is weaker than $\alpha[\phi]$.

So it follows that if $\alpha[\phi]$ is defined but $\alpha[\psi]$ is not then there is no adequate rewrite rule for $\alpha \wedge \beta$ when the condition on the right-hand side is not met. By symmetry, the same follows if $\alpha[\psi]$ is defined but $\alpha[\phi]$ is not. (The case where $\alpha[\psi]$ and $\alpha[\phi]$ are undefined follows immediately from either of the symmetric cases.) That concludes the proof.

Negation Suppose $\alpha[\phi]$ is not defined. Then we can show by induction that no rewrite rule will be both defined and adequate for negation. We can show this by showing that every rewrite rule for $\alpha[\neg \phi]$ that is defined is either logically equivalent to a contradiction (e.g. $\alpha \backslash \alpha$ ) or to $\alpha$ itself.

Disjunction This follows from the discussions of disjunction and negation and the fact that $\alpha[\phi \vee \psi]$ is equivalent to $\alpha[\neg(\neg \phi \wedge \neg \psi)]$.

Conditional This follows from the discussions of disjunction and negation and the fact that the conditional is equivalent to $\alpha[\neg \phi \vee \psi]$.

Proposition 2. Suppose $\phi$ and $\psi$ are CCPS with monotonic definedness conditions and intersective meanings and $\alpha$ is a sentence. It follows on the semantics of (27) that the projection properties match those of Heim's system, listed here:

- $\alpha[\neg \phi]$ is defined iff $\alpha \backslash \alpha[\phi]$ is defined.

- $\alpha[\phi \wedge \psi]$ is defined iff $(\alpha[\phi])[\psi]$ is defined.

- $\alpha[\phi \vee \psi]$ is defined iff $(\alpha[\neg \phi])[\psi]$ is defined.

- $\alpha[\phi \rightarrow \psi]$ is defined iff $(\alpha[\phi])[\psi]$ is defined. 
Proof. The right-to-left direction follows immediately from the proposed rewrite rules in the proof of Proposition 1. For the left-to-right direction: Negation is unaffected by the order rule since it only takes one argument, so the proof above still works. For the rest of the connectives the proofs follow from minor modification of the proofs in Proposition 1. For instance, for conjunction we can eliminate the possibility that $\alpha[\phi]$ is undefined, since if it were we could not introduce $\phi$ in the rewrite rule in any way (except to add it to $\alpha \backslash \alpha$, where it would have no effect), thus there will be no adequate rewrite rule. Once we eliminate that possibility the limited definedness conditions follow.

Proposition 3. For a quantifier $Q$ satisfying both the Conservativity and NonTriviality, CCPs $\phi$ and $\psi$, variable $x$, and sentence $\alpha, \alpha\left[Q_{i}(\phi, \psi)\right]$ is defined iff $\alpha[\phi]$ is defined and $(\alpha[\phi])[\psi]$ is defined.

Proof. I give only a sketch here. The existence of the rewrite rule is easy to prove: We just use: $\alpha\left[Q_{i}(\phi, \psi)\right]=\alpha \wedge Q_{i}(\alpha[\phi], \alpha[\phi][\psi])$. Given the order constraint and non-triviality it is clear that if $\alpha[\phi]$ is undefined there is no way of producing a defined truth-conditionally adequate rewrite rule, given non-triviality. So it must be defined. What about $(\alpha[\phi])[\psi]$ ? If it is undefined, then so is $\alpha[\psi]$, given the non-monoticity of the CCPs. But there is no truth-conditionally adequate rewrite rule for $\alpha\left[Q_{i}(\phi, \psi)\right]$ that does not make use of $\alpha[\psi]$ or $(\alpha[\phi])[\psi]$ because of the non-triviality assumption.

\section{References}

Beaver, David. 1992. The kinematics of presupposition. Amsterdam Colloquium 8. https://webspace.utexas.edu/dib97/tkop.pdf.

Beaver, David. 1994. When variables don't vary enough. Semantics and Linguistic Theory (SALT) 4. 35-6o. https://webspace.utexas.edu/dib97/ salt4.pdf.

Beaver, David. 2001. Presupposition and assertion in dynamic semantics. CSLI. https://webspace.utexas.edu/dib97/silli.pdf.

Beaver, David. 2008. As brief as possible (but not briefer). Theoretical Linguistics 34(3). 213-228. doi:10.1515/THLI.2008.014.

Beaver, David \& Bart Geurts. 2011. Presupposition. In Edward N. Zalta (ed.), The Stanford encyclopedia of philosophy, Summer 2011 edn. http: //plato.stanford.edu/entries/presupposition/. 
Explaining presupposition projection

Breheny, Richard. 2003. Exceptional scope indefinites and domain restriction. Sinn und Bedeutung 7. 38-52. http://ling.sprachwiss.uni-konstanz.de/ pages/conferences/sub7/proceedings/download/sub7_breheny.pdf.

Charlow, Simon. 2009. "Strong" predicative presuppositional objects. Slides from a talk at European Summer School in Logic, Language and Information (ESSLLI). http://homepages.nyu.edu/ sec392/handouts/esslli_ presup.pdf.

Chemla, Emmanuel. 2008. Similarity: Towards a unified account of scalar implicatures, free choice permission and presupposition projection. Unpublished manuscript, ENS. http://semanticsarchive.net/Archive/WI1ZTU3N/ Chemla-SIandPres.html.

Chemla, Emmanuel. 2009. Presuppositions of quantified sentences: Experimental data. Natural Language Semantics 17(4). 299-340. doi:10.1007/s11050-009-9043-9.

Chemla, Emmanuel \& Philippe Schlenker. 2009. Incremental vs. symmetric acounts of presupposition projection: An experimental approach. Ms. IJN, LSCP, \& NYU. http://semanticsarchive.net/Archive/mU4YjY5Y/ Chemla-Schlenker-Symmetry.html.

Edgington, Dorothy. 1995. On conditionals. Mind 104(414). 235-329. doi:10.1093/mind/104.414.235.

von Fintel, Kai. 1994. Restrictions on quantifier domains. Amherst, MA: University of Massachusetts dissertation. http://www.semanticsarchive. net/Archive/jA3N2IwN/fintel-1994-thesis.pdf.

Fox, Danny. 2008. Two short notes on Schlenker's theory of presupposition projection. Theoretical Linguistics 34(3). 237-252. doi:10.1515/THLI.2008.016.

Gawron, Jean Mark. 1996. Quantification, quantificational domains, and dynamic logic. In Shalom Lappin (ed.), The handbook of contemporary semantic theory, 246-267. Blackwell.

Gazdar, Gerald. 1979. Pragmatics: Implicature, presupposition and logical form. Academic Press.

George, Benjamin. 2007. Predicting presupposition projection: some alternatives in the strong Kleene tradition. Manuscript, UCLA. http: //semanticsarchive.net/Archive/DYoYTgxN/.

Geurts, Bart. 1996. Local satisfaction guaranteed: A presupposition theory and its problems. Linguistics and Philosophy 19(3). 259-294. doi:10.1007/BFoo628201.

Geurts, Bart \& Rob van der Sandt. 1999. Domain restriction. In Peter Bosch \& 
Rob van der Sandt (eds.), Focus: Linguistic, cognitive and computational perspectives, 268-292. Cambridge University Press. http://phil.ruhosting. $\mathrm{nl} / \mathrm{tfl} / \mathrm{bart} /$ papers/domainrestriction.pdf.

Heim, Irene. 1982. The semantics of definite and indefinite noun phrases. Amherst: University of Massachusetts dissertation. http: //semanticsarchive.net/Archive/TkoZmYyY/.

Heim, Irene. 1983a. File change semantics and the familiarity theory of definiteness. In Rainer Bäuerle, Christoph Schwarze \& Arnim von Stechow (eds.), Meaning, use, and interpretation of language, 164-19o. Walter de Gruyter.

Heim, Irene. 1983b. On the projection problem for presuppositions. West Coast Conference on Formal Linguistics 2. 114-125.

Heim, Irene. 1990. Presupposition projection. In Rob van der Sandt (ed.), Reader for the Nijmegen workshop on presupposition, lexical meaning, and discourse processes, University of Nijmegen. http://semanticsarchive. net/Archive/GFiMGNjN/.

Horn, Laurence. 1972. The semantics of the logical operators in English. Los Angeles, CA: UCLA dissertation.

Karttunen, Lauri. 1973. Presuppositions of compound sentences. Linguistic Inquiry 4(2). 169-93. http://www.jstor.org/stable/4177763.

Karttunen, Lauri. 1974. Presupposition and linguistic context. Theoretical Linguistics 1(1-3). 181-93. doi:10.1515/thli.1974.1.1-3.181.

Kratzer, Angelika. 1986. Conditionals. Chicago Linguistics Society 22(2). 1-15.

Levinson, Stephen. 200o. Presumptive meanings: The theory of generalized conversational implicatures. Cambridge, MA: MIT Press.

Rothschild, Daniel. 2007. The elusive scope of descriptions. Philosophy Compass 2(6). 910-927. doi:10.1111/j.1747-9991.2007.00111.x.

Rothschild, Daniel. 2008a. Presupposition projection and logical equivalence. Philosophical Perspectives 22(1). 473-497. doi:10.1111/j.15208583.2008.00154.x.

Rothschild, Daniel. 2008b. Transparency theory and its dynamic alternatives. Theoretical Linguistics 34(3). 261-268. doi:10.1515/THLI.2008.018.

van der Sandt, Rob. 1992. Presupposition projection as anaphora resolution. Journal of Semantics 9(4). 333-377. doi:10.1093/jos/9.4.333.

Schlenker, Philippe. 2006. Anti-dynamics: Presupposition projection without dynamic semantics. Journal of Logic, Language, and Information 16(3). 325-56. doi:10.1007/s10849-006-9034-X.

Schlenker, Philippe. 2008a. Be articulate: A pragmatic theory of 
Explaining presupposition projection

presupposition projection. Theoretical Linguistics 34(3). 157-212. doi:10.1515/THLI.2008.013.

Schlenker, Philippe. 2008b. Local contexts. Unpublished early version of Schlenker 2009. https://files.nyu.edu/pds4/public/Local_Contexts_Old. pdf.

Schlenker, Philippe. 2008c. Presupposition projection: Explanatory strategies. Theoretical Linguistics 34(3). 287-316. doi:10.1515/THLI.2008.021.

Schlenker, Philippe. 2008d. Presupposition projection: The new debate. Semantics and Linguistic Theory (SALT) 18. 655-693. http://hdl.handle. net/1813/13061.

Schlenker, Philippe. 2009. Local contexts. Semantics and Pragmatics 2(3). 1-78. doi:10.3765/sp.2.3.

Schlenker, Philippe. 2010. Local contexts and local meanings. Philosophical Studies 151(1). 115-142. doi:10.1007/s11098-010-9586-o.

Schwarzschild, Roger. 2002. Singleton indefinites. Journal of Semantics 19(3). 289-314. doi:10.1093/jos/19.3.289.

Soames, Scott. 1982. How presuppositions are inherited: A solution to the projection problem. Linguistic Inquiry 13(3). 483-545. http://www.jstor. org/stable/4178288.

Soames, Scott. 1989. Presuppositions. In D. Gabbay \& F. Guenther (eds.), Handbook of philosophical logic, vol. IV, 553-616. Dordrecht.

Stalnaker, Robert. 1973. Presuppositions. Journal of Philosophical Logic 2(4). 447-457. doi:10.1007/BFoo262951.

Stalnaker, Robert. 1974. Pragmatic presuppositions. In Milton K. Munitz \& Peter K. Unger (eds.), Semantics and philosophy, 197-213. New York: New York University Press.

Stalnaker, Robert. 2010. Responses to Stanley and Schlenker. Philosophical Studies 151(1). 143-147. doi:10.1007/s11098-010-9587-Z.

Veltman, Frank. 1996. Defaults in update semantics. Journal of Philosophical Logic 25(3). 221-261. doi:10.1007/BFoo248150.

Daniel Rothschild

All Souls College

Oxford

$\mathrm{OX} 14 \mathrm{AL}$

UK 